\title{
A note on tight cuts in matching-covered graphs
}

\author{
Xiao Zhao ${ }^{1 *}$ \\ Sheng Chen ${ }^{2}$ \\ 1 College of Mathematics and Information Science, Henan Normal University, Xinxiang, China \\ 2 Department of Mathematics, Harbin Institute of Technology, Harbin, China
}

received $8^{\text {th }}$ Jan. 2020, revised $17^{\text {th }}$ June 2020, 19 ${ }^{\text {th }}$ Jan. 2021, $28^{\text {th }}$ May 2021, accepted $28^{\text {th }}$ May 2021.

\begin{abstract}
Edmonds, Lovász, and Pulleyblank showed that if a matching covered graph has a nontrivial tight cut, then it also has a nontrivial ELP-cut. Carvalho et al. gave a stronger conjecture: if a matching covered graph has a nontrivial tight cut $C$, then it also has a nontrivial ELP-cut that does not cross $C$. Chen, et al gave a proof of the conjecture. This note is inspired by the paper of Carvalho et al. We give a simplified proof of the conjecture, and prove the following result which is slightly stronger than the conjecture: if a nontrivial tight cut $C$ of a matching covered graph $G$ is not an ELP-cut, then there is a sequence $G_{1}=G, G_{2}, \ldots, G_{r}, r \geq 2$ of matching covered graphs, such that for $i=1,2, \ldots, r-1, G_{i}$ has an ELP-cut $C_{i}$, and $G_{i+1}$ is a $C_{i}$-contraction of $G_{i}$, and $C$ is a 2-separation cut of $G_{r}$.
\end{abstract}

Keywords: matching covered graph, tight cut, ELP-cut, 2-separation cut

\section{Introduction}

For graph theoretical terminology and notation, we follow Bondy and Murty (2008). For the terminology that is specific to matching covered graphs, we follow Lovász (1987). This article studies finite and undirected loopless graphs. A perfect matching of graph $G$ is a set of independent edges which covers all vertices of $G$. An edge of graph $G$ is admissible if there is a perfect matching of the graph which contains it. A nontrivial graph is matchable if it has at least one perfect matching, and is matching covered if it is connected and each of its edges is admissible. A nontrivial graph $G$ is critical if $G-v$ is matchable for any $v \in V(G)$, is bicritical if $G-u-v$ has a perfect matching for any two distinct vertices $u$ and $v$ of $G$.

For $X \subseteq V, \bar{X}=V-X$ is the complement of $X$. The set of all edges of $G$ with exactly one end in $X$ is denoted by $\partial(X)$, and is referred to as a cut of $G$. We call $X$ and $\bar{X}$ the shores of $\partial(X)$. For any cut $C:=\partial(X)$ of a graph $G$, we denote the graph obtained from $G$ by shrinking the shore $X$ to a single vertex $x$ by $G /(X \rightarrow x)$. Let $G / X$ and $G / \bar{X}$ be obtained from $G$ by contracting $X$ and $\bar{X}$, respectively, and call them $\partial(X)$-contractions of $G$. We call an edge cut $\partial(X)$ trivial if $|X|=1$ or $|\bar{X}|=1$. An edge cut $C:=\partial(X)$ of $G$ is called a tight cut if $|C \cap M|=1$ for each perfect matching $M$ of $G$. A matching covered graph which is free of nontrivial tight cuts is a brick if it is nonbipartite. If $C:=\partial(X)$ is a tight cut of a matching covered graph $G$, then both the $C$-contractions of $G$ are also matching covered. Two

${ }^{*}$ Corresponding author. Email: zhaoxiao05@126.com

ISSN 1365-8050 @ 2021 by the author(s) Distributed under a Creative Commons Attribution 4.0 International License 
cuts $C=\partial(X)$ and $\partial(Y)$ of a matching covered graph cross if each of the four sets $X \cap Y, X \cup \bar{Y}$, $\bar{X} \cap Y$, and $\bar{X} \cap \bar{Y}$ is nonempty. Thus, if $C$ and $D$ do not cross, then one of the two shores of $C$ is a subset of one of the two shores of $D$.

A barrier in a matchable graph $G$ is a subset $B$ of $V$ for which $o(G-B)=|B|$, where $o(G-B)$ is the number of odd components of graph $G-B$. A barrier is trivial if it is a singleton. If $B$ is a barrier and $H$ is any odd connected component of $G-B$, then it is easy to show that $\partial(V(H))$ is tight. Such cuts are called barrier cuts. Note that if $B$ is a barrier of matching covered graph $G$, then there is no even component in $G-B$ and $B$ is independent. Two distinct vertices $\{u, v\}$ of matching covered graph $G$ is a 2-separation if $G=G_{1} \cup G_{2}$ and $V\left(G_{1}\right) \cap V\left(G_{2}\right)=\{u, v\}$ and $\left|V\left(G_{i}\right)\right|$ is even for $i=1,2$. It can be verified that both $\partial\left(V\left(G_{1}\right)-u\right)$ and $\partial\left(V\left(G_{2}\right)-v\right)$ are tight cuts of $G$. Such cuts are called 2-separation cuts. Barrier-cuts and 2-separation cuts are called ELP-cuts.

Let $G$ be a matchable graph, and $B$ be a nonempty barrier of $G$. The bipartite graph $H(B)$ obtained from $G$ by deleting the vertices in the even components of $G-B$, contracting every odd component to a single vertex, and deleting the edges with both ends in $B$, is called the core of $G$ with respect to the barrier $B$. Barrier $B$ of $G$ is a $D M$-barrier if each odd component of $G-B$ is critical, and the core $H(B)$ of $G$ with respect to $B$ is matching covered.

Carvalho et al. (2018) proved the following important result about DM-barrier.

Lemma 1. (Carvalho et al. (2018)) Let $G$ be a matchable graph, and let $X$ be a nonempty proper subset of $V(G)$ such that both the subgraphs $G[X]$ and $G[\bar{X}]$ are connected, and no edge in the cut $\partial(X)$ is admissible in $G$. Then $G$ has a DM-barrier $B$ which is a subset of $X$ or of $\bar{X}$. Furthermore, the vertex sets of all the odd components of $G-B$ are also subsets of that same shore.

Edmonds et al. (1982) proved the ELP Theorem: if a matching covered graph has a nontrivial tight cut, then it also has a nontrivial ELP-cut. A purely graph theoretical proof was given by Szigeti (2002). Carvalho et al. (2002) provided an alternative proof of the ELP Theorem by using Lemma 1, and gave the following conjecture.

Conjecture 2. (Carvalho et al. (2018)) Let $C$ be a nontrivial tight cut of a matching covered graph $G$. Then, $G$ has an ELP-cut that does not cross $C$.

Carvalho et al. (2018) established the validity of Conjecture 2 for bicritical graphs and matching covered graphs with at most two bricks. Also, they observed that Conjecture 2 is rephrased as follow: let $C$ be a nontrivial tight cut of a matching covered graph $G$, then there is a sequence $G_{1}=G, G_{2}, \ldots, G_{r}, r \geq 1$ of matching covered graphs, such that for $i=1,2, \ldots, r-1, G_{i}$ has an ELP-cut $C_{i}, G_{i+1}$ is a $C_{i}$-contraction of $G_{i}$, and $C$ is an ELP-cut of $G_{r}$. Chen et al. (2020) gave a proof of Conjecture 2 in a preprint.

Inspired by the proof of Carvalho et al. of the ELP Theorem, we give a simplified proof of Conjecture 2 and prove a slightly stronger result:

Theorem 3. If a nontrivial tight cut $C$ of a matching covered graph $G$ is not an ELP-cut, then there is a sequence $G_{1}=G, G_{2}, \ldots, G_{r}, r \geq 2$ of matching covered graphs, such that for $i=1,2, \ldots, r-1, G_{i}$ has an ELP-cut $C_{i}, G_{i+1}$ is a $C_{i}$-contraction of $G_{i}$, and $C$ is a 2 -separation cut of $G_{r}$.

\section{Preliminary}

The following basic results of matching covered graphs will be used in the proof of our main theorem in the next section. 
Lemma 4. (Plummer (1980)) Every matching covered graph is 2-connected except $K_{2}$.

Lemma 5. (Carvalho et al. (2018)) Let $G$ be a matching covered graph, and let $C$ be a tight cut of $G$. Then both $C$-contractions are matching covered. Moreover, if $G^{\prime}$ is a $C$-contraction of $G$ then a tight cut of $G^{\prime}$ is also a tight cut of $G$. Conversely, if a tight cut of $G$ is a cut of $G^{\prime}$ then it is also tight in $G^{\prime}$.

Lemma 6. Let $B$ be a nontrivial barrier of a matching covered graph $G$, and $G[Y]$ be an odd component of $G-B$. Suppose that $G^{\prime}=G /(\bar{Y} \rightarrow \bar{y})$, and $B^{\prime}$ is a barrier of $G^{\prime}$. If $\bar{y} \in B^{\prime}$, then $B \cup\left(B^{\prime}-\bar{y}\right)$ is a barrier of $G$, and every component of $G^{\prime}-B^{\prime}$ is also a component of $G-\left(B \cup\left(B^{\prime}-\bar{y}\right)\right)$, otherwise $B^{\prime}$ is also a barrier of $G$.

Proof: Let $H_{1}, H_{2}, \ldots, H_{|B|-1}, Y$ be all odd components of $G-B$, and $O_{1}, O_{2}, \ldots, O_{\left|B^{\prime}\right|}$ be all odd components of $G^{\prime}-B^{\prime}$. Since $Y$ is an odd component of $G-B$, every edge that is incident with $\bar{y}$ in $G^{\prime}$ is incident with one vertex of $B$ in $G$. If $\bar{y} \in B^{\prime}$, then $B \cup\left(B^{\prime}-\bar{y}\right)$ is a barrier of $G$ and $H_{1}, H_{2}, \ldots, H_{|B|-1}, O_{1}, O_{2}, \ldots, O_{\left|B^{\prime}\right|}$ are all odd components of $G-\left(B \cup\left(B^{\prime}-\bar{y}\right)\right)$. If $\bar{y} \notin B^{\prime}$, adjust notation so that $\bar{y} \in O_{1}$. By Lemmas 4 and $5, G[\bar{Y}]$ is connected. Then $B^{\prime}$ is also a barrier of $G$ and $G\left[V\left(O_{1}-\bar{y}\right) \cup \bar{Y}\right], O_{2}, \ldots, O_{\left|B^{\prime}\right|}$ are all odd components of $G-B^{\prime}$ since

Lemma 7. Let $\{u, v\}$ be a 2-separation of matching covered graph $G$ which gives rise to 2-separation cut $D:=\partial(Y)$. Adjust notation so that $u \in Y$ and $v \in \bar{Y}$. Suppose that $G^{\prime}=G /(\bar{Y} \rightarrow \bar{y})$, and $B$ is a barrier of $G^{\prime}$. If $\bar{y} \in B$, then $(B-\bar{y})+v$ is a barrier of $G$. If $\bar{y} \notin B$, then $B$ is a barrier of $G$.

Proof: If $\bar{y} \notin B$, then it is obvious that $B$ is a barrier of $G$. If $\bar{y} \in B$, then $u \notin B$ since $\bar{y} u \in E\left(G^{\prime}\right)$ and $G^{\prime}$ is matching covered graph. Let $L$ be a component of $G^{\prime}-B$ that contains vertex $u$. We conclude that except $\bar{y} u$, every edge that is incident with $\bar{y}$ in $G^{\prime}$ is incident with $v$ in $G$. Hence every component of $G^{\prime}-B$ is also a component of $G-((B-\bar{y})+v)$ except $L$. It is easy to see that $G[V(L) \cup(\bar{Y}-v)]$ is an odd component of $G-((B-\bar{y})+v)$. Hence $(B-\bar{y})+v$ is also a barrier of $G$.

\section{Proof of the main result}

In this section, the proof of Lemma 8 uses arguments similar to that of Theorem 4.1 of Carvalho et al. (2018). The statement of Lemma 9 is the same as Theorem 1.11 of Chen et al. (2020).

Lemma 8. Let $C:=\partial(X)$ be a nontrivial tight cut of a matching covered graph $G$. If there is an edge $e:=u v \in C$ such that $u \in X, v \in \bar{X}$ and both $G[X-u]$ and $G[\bar{X}-v]$ are connected, then $G$ has $a$ nontrivial barrier that is a proper subset of $X$ or $\bar{X}$, or has a nontrivial 2-separation cut that does not cross $C$.

Proof: By Lemmas 4 and $5, G /(X \rightarrow x)$ and $G /(\bar{X} \rightarrow \bar{x})$ are 2-connected, whence both $G[X]$ and $G[\bar{X}]$ are connected. The analysis is divided into the following two cases.

Case 1: $v$ is the only neighbor of $u$ in $\bar{X}$ and $u$ is the only neighbor of $v$ in $X$.

Let $G^{\prime}:=G-u-v$. Since $G$ is a matching covered graph, $G$ has a perfect matching $M$ that contains edge $e$. Then $M-e$ is a perfect matching of $G^{\prime}$, and we deduce that $G^{\prime}$ has perfect matchings. Moreover, $C-e=\partial_{G^{\prime}}(X-u)=\partial_{G^{\prime}}(\bar{X}-v)$ is a cut of $G^{\prime}$ and no edge of $C-e$ is admissible in $G^{\prime}$.

By Lemma $1, G^{\prime}$ has a DM-barrier $B^{\prime}$ such that $B^{\prime}$, as well as the vertex sets of all the odd components $O_{1}, O_{2}, \ldots, O_{\left|B^{\prime}\right|}$ of $G^{\prime}-B^{\prime}$, are subsets of one of $X-u$ and $\bar{X}-v$. Adjust notation so that $B^{\prime} \subsetneq X-u$ 
and $O_{i} \subsetneq X-u$ for $i \in\left\{1,2, \cdots,\left|B^{\prime}\right|\right\}$. Since $G[\bar{X}-v]$ is connected, $\bar{X}-v$ is a subset of the vertex set of an even component, say $L$, of the graph $G^{\prime}-B^{\prime}$.

Let $B:=B^{\prime} \cup\{u\}$. By hypothesis, $u$ is the only vertex of $X$ adjacent to $v$. Thus, $O_{1}, O_{2}, \ldots, O_{\left|B^{\prime}\right|}$ and $G[V(L)+v]$ are also odd components of $G-B$, implying that $B$ is a nontrivial barrier of $G$ and $B \subsetneq X$.

Case 2: $u$ has two or more neighbours in $\bar{X}$, or $v$ has two or more neighbours in $X$.

Without loss of generality, we assume that $u$ has two or more neighbours in $\bar{X}$. Let $R:=\partial(u) \backslash C$. Now consider the graph $G^{\prime \prime}:=G-R$, together with the cut $D:=\partial(X-u)$ in $G^{\prime \prime}$. Since both $G[X-u]$ and $G[\bar{X}]$ are connected, the graphs $G^{\prime \prime}[X-u]$ and $G^{\prime \prime}[\bar{X}+u]$ are both connected.

Every perfect matching of $G$ containing edge $e$ is also a perfect matching of $G^{\prime \prime}$. Thus, $G^{\prime \prime}$ has perfect matchings. Since $u$ is not adjacent to any vertex of $X$ in $G^{\prime \prime}$ and $C$ is a tight cut of $G$, no edge of $D$ is admissible in $G^{\prime \prime}$. By Lemma $1, G^{\prime \prime}$ has a DM-barrier $B^{\prime \prime}$ such that $B^{\prime \prime}$, as well as the vertex sets of all the odd components $H_{1}, H_{2}, \ldots, H_{\left|B^{\prime \prime}\right|}$ of $G^{\prime \prime}-B^{\prime \prime}$, are subsets of one of $X-u$ and $\bar{X}+u$.

If $V\left(H_{1}\right), V\left(H_{2}\right), \ldots, V\left(H_{\left|B^{\prime \prime}\right|}\right)$ and $B^{\prime \prime}$ are proper subsets of $X-u$, then $\bar{X}+u$ is a subset of the vertex set of an even component, say $L_{1}$, of the graph $G^{\prime \prime}-B^{\prime \prime}$ since $G[\bar{X}+u]$ is connected. Hence $B:=B^{\prime \prime} \cup\{u\}$ is a nontrivial barrier of $G$, and $H_{1}, H_{2}, \ldots, H_{\left|B^{\prime \prime}\right|}$ and $G\left[V\left(L_{1}\right)-u\right]$ are all odd components of $G-B$. As $B^{\prime \prime} \subsetneq X-u$ and $u \in X, B \subsetneq X$.

If $V\left(H_{1}\right), V\left(H_{2}\right), \ldots, V\left(H_{\left|B^{\prime \prime}\right|}\right)$ and $B^{\prime \prime}$ are proper subsets of $\bar{X}+u$, then $X-u$ is a subset of the vertex set of an even component, say $L_{2}$, of the graph $G^{\prime \prime}-B^{\prime \prime}$ since $G[X-u]$ is connected. Then $u$ does not lie in $B^{\prime \prime}$. Otherwise, $G^{\prime \prime}-B^{\prime \prime}=G-B^{\prime \prime}$ and $B^{\prime \prime}$ is a barrier of $G$. Further, $L_{2}$ is also an even component of $G-B^{\prime \prime}$, contradicting the condition that $G$ is matching covered. Hence $u$ is a vertex of an even component of $G^{\prime \prime}-B^{\prime \prime}$ or $u$ is a vertex of an odd component of $G^{\prime \prime}-B^{\prime \prime}$.

Subcase 2.1: $u$ is a vertex of an even component of $G^{\prime \prime}-B^{\prime \prime}$, say $L_{3}$.

Then $L_{2}=L_{3}$. Otherwise, $H_{1}, H_{2}, \ldots, H_{\left|B^{\prime \prime}\right|}$ and $G\left[V\left(L_{3}\right)-u\right]$ are all odd components of $G-$ ( $B^{\prime \prime} \cup\{u\}$ ), implying that $B^{\prime \prime} \cup\{u\}$ is a nontrivial barrier of $G$. $L_{2}$ is an even component of $G^{\prime \prime}-B^{\prime \prime}$, implying that $L_{2}$ is also an even component of $G-\left(B^{\prime \prime} \cup\{u\}\right)$, contradicting the condition that $G$ is matching covered. Hence $L_{2}=L_{3}$. Since $u$ is not adjacent to any vertex of $X-u$ in $G^{\prime \prime}$ and $\left|V\left(L_{2}\right)\right|$ is even, $V\left(L_{2}\right) \cap \bar{X} \neq \emptyset$. For any $w \in V\left(L_{2}\right) \cap \bar{X}, B:=B^{\prime \prime} \cup\{w\}$ is a nontrivial barrier of $G$, and $H_{1}, H_{2}, \ldots, H_{\left|B^{\prime \prime}\right|}$ and $G\left[V\left(L_{2}\right)-w\right]$ are all odd components of $G-B$. As $B^{\prime \prime} \subsetneq \bar{X}$ and $w \in \bar{X}$, $B \subsetneq \bar{X}$.

Subcase 2.2: $u$ is a vertex of an odd component of $G^{\prime \prime}-B^{\prime \prime}$.

Adjust notation so that $u \in V\left(H_{1}\right)$. Note that $u$ is the only vertex in an odd component of $G^{\prime \prime}-B^{\prime \prime}$ that is adjacent to vertices of $X$ in $G$. Then the barrier $B^{\prime \prime}$ of $G^{\prime \prime}$ is also a barrier of $G$, and $H_{2}, \ldots, H_{\left|B^{\prime \prime}\right|}$ and $G\left[V\left(H_{1}\right) \cup V\left(L_{2}\right)\right]$ are all odd components of $G-B^{\prime \prime}$. If $B^{\prime \prime}$ is nontrivial, we are done with this proof.

If $B^{\prime \prime}$ is trivial, $u$ lies in $V\left(H_{1}\right)$ and has at least two neighbours in $G^{\prime \prime}$. Thus there is also at least one neighbour of $u$ lying in $V\left(H_{1}\right)$, whence $\left|V\left(H_{1}\right)\right| \geq 2$. As $H_{1}$ is an odd component of $G^{\prime \prime}-B^{\prime \prime}$, $\left|V\left(H_{1}\right)-u\right|$ is even. Let $z$ denote the only vertex of $B^{\prime \prime}$. We have $\{u, z\}$ is a 2-separation of $G$, and $\partial\left(\left(V\left(H_{1}\right)-u\right)+z\right)$ is a 2-separation cut of $G$. As $\left(V\left(H_{1}\right)-u\right)+z \subseteq \bar{X}, \partial\left(\left(V\left(H_{1}\right)-u\right)+z\right)$ does not cross $C$.

Remark 1: In the proof of Subcase 2.2 of Lemma 8, if $B^{\prime \prime}=\{z\}$ and $G[\bar{X}+u]$ is 2-connected, then $G[\bar{X}+u-z]$ is connected. As $G^{\prime \prime}[\bar{X}+u-z]=G[\bar{X}+u-z], G^{\prime \prime}[\bar{X}+u-z]$ is connected and 
$H_{1}=G^{\prime \prime}[\bar{X}+u-z]$, implying that $\{u, z\}$ is a 2-separation of $G$, and $\partial(\bar{X})$ is a 2-separation cut of $G$, i.e. $C$ is a 2-separation cut of $G$. Hence under the condition of Lemma 8 , if $G[\bar{X}+u]$ is 2-connected, then $G$ has a nontrivial barrier that is a proper subset of $X$ or $\bar{X}$, or $C$ is 2-separation cut of $G$ that arises from 2-separation $\{u, z\}$, where $z \in \bar{X}$.

Lemma 9. Let $C:=\partial(X)$ be a nontrivial tight cut of a matching covered graph $G$. Then $G$ has a nontrivial barrier $B$ of $G$ that is a proper subset of $X$ or $\bar{X}$, or has a nontrivial 2-separation cut that does not cross $C$.

Proof: If there is an edge $e:=u v \in C$ such that $u \in X, v \in \bar{X}$ and both $G[X-u]$ and $G[\bar{X}-v]$ are connected, then by Lemma 8 , the result holds.

Now suppose that there is no edge $e:=u v \in C$, with $u \in X$ and $v \in \bar{X}$, such that both $G[X-u]$ and $G[\bar{X}-v]$ are connected. Then for any edge $e:=u v \in C$, with $u \in X$ and $v \in \bar{X}, u$ is a cut vertex of $G[X]$ or $v$ is a cut vertex of $G[\bar{X}]$. If $G[X]$ has no cut vertex, then every vertex of $G[\bar{X}]$ that is incident with an edge of $C$ is a cut vertex of $G[\bar{X}]$ by hypothesis, implying that some cut vertex of $G[\bar{X}]$ is also a cut vertex of $G$. This contradicts that $G$ is matching covered graph. Hence $G[X]$ has two or more blocks. Likewise, $G[\bar{X}]$ has two or more blocks.

Since $G$ is finite and 2-connected, there is a cut vertex $v$ of $G[X]$ which is incident with an edge of $C$, such that one component $F_{1}$ of $G[X]-v$ has no cut vertex of $G[X]$ that is incident with an edge of $C$. Let $F_{2}:=G\left[X \backslash V\left(F_{1}\right)\right]$, as shown in Figure 1 .

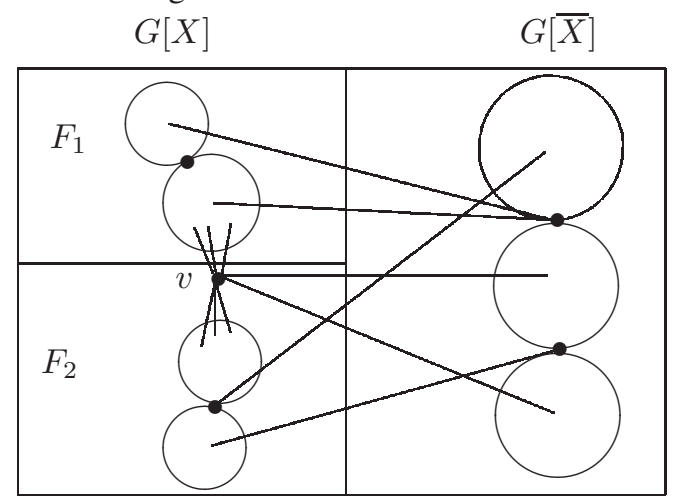

Fig. 1: The component $F_{1}$ of $G[X]-v$

As $v$ is a cut vertex of $G[X], G /(\bar{X} \rightarrow \bar{x})-\{\bar{x}, v\}$ is not connected. If $o(G /(\bar{X} \rightarrow \bar{x})-\{\bar{x}, v\})>0$, then $o(G /(\bar{X} \rightarrow \bar{x})-\{\bar{x}, v\})=|\{\bar{x}, v\}|=2$ since $G /(\bar{X} \rightarrow \bar{x})$ is matching covered graph, i.e., $\{\bar{x}, v\}$ is a barrier of $G /(\bar{X} \rightarrow \bar{x})$ and $\bar{x} v \notin E(G /(\bar{X} \rightarrow \bar{x}))$. This contradicts that $v$ is incident with an edge of $C$. Hence $o(G /(\bar{X} \rightarrow \bar{x})-\{\bar{x}, v\})=0$, implying that $\{\bar{x}, v\}$ is a 2-separation of $G /(\bar{X} \rightarrow \bar{x})$.

Hence $\partial\left(V\left(F_{2}\right)\right)$ is a 2-separation cut of $G /(\bar{X} \rightarrow \bar{x})$. By Lemma 5, $\partial\left(V\left(F_{2}\right)\right)$ is also a tight cut of $G$. Let $G_{2}:=G /\left(V\left(F_{2}\right) \rightarrow s\right)$, as shown in Figure 2. By Lemma 5, $C$ is a nontrivial tight cut of $G_{2}$ and $V\left(F_{1}\right)+s$ and $\bar{X}$ are two shores of $C$.

If $G_{2}[\bar{X}+s]$ has two or more blocks, there is a block $K$ that satisfies $s \notin V(K)$ and contains precisely one cut vertex, say $k$, of $G_{2}[\bar{X}+s]$. Note that $s$ is not cut vertex of $G_{2}[\bar{X}+s]$ since $G_{2}[\bar{X}]$ is connected. By the definition of $F_{1}$, every edge of $C$ that is incident with a vertex of $F_{1}$ is incident with a cut vertex 


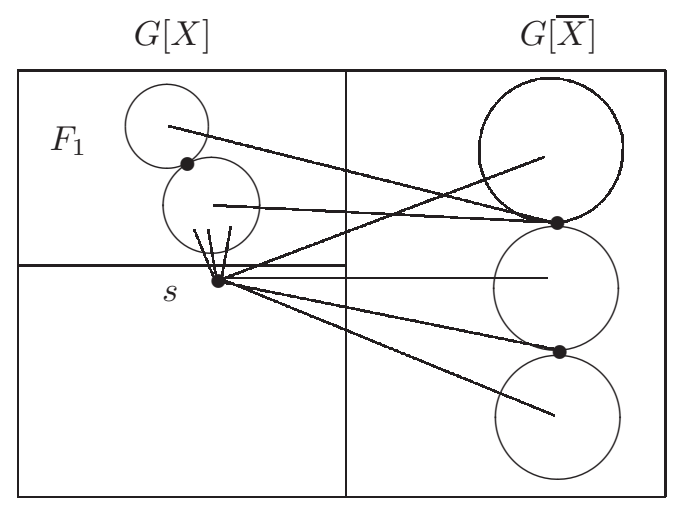

Fig. 2: Graph $G_{2}:=G /\left(V\left(F_{2}\right) \rightarrow s\right)$

of $G_{2}[\bar{X}]$, implying that $k$ is also a cut vertex of $G_{2}$. This contradicts that $G_{2}$ is matching covered graph. Hence $G_{2}[\bar{X}+s]$ is 2-connected.

Since $G_{2}[\bar{X}+s]$ is 2-connected, there is a vertex $w$ of $\bar{X}$ which is not a cut vertex of $G[\bar{X}]$ and adjoins $s$. It is easy to see that $s w \in C$ and both $G_{2}[\bar{X}-w]$ and $F_{1}$ are connected. By Remark $1, G_{2}$ has a nontrivial barrier $B$ satisfying $B \subsetneq \bar{X}$ or $B \subsetneq V\left(F_{1}\right)+s$, or $C$ is 2-separation cut of $G_{2}$ that arises from 2 -separation $\{s, z\}$, where $z \in \bar{X}$. Next, two cases should be considered.

Case 1: There is a nontrivial barrier $B$ in $G_{2}$, where $B \subsetneq \bar{X}$ or $B \subsetneq V\left(F_{1}\right)+s$.

If $s \notin B$, then $B$ is also a barrier of $G$. If $s \in B$, then $B \subsetneq V\left(F_{1}\right)+s$, implying that $\bar{X}$ is a subset of the vertex set of an odd component, say $L$, of $G_{2}-B$. Since $v$ is a cut vertex of $G[X]$, every edge of $G_{2}\left[V\left(F_{1}\right)+s\right]$ that is incident with $s$ is incident with $v$ in $G$. Hence $B^{\prime}:=(B-s)+v$ is a barrier of $G, G\left[V(L) \cup V\left(F_{2}-v\right)\right]$ is an odd component of $G-B^{\prime}$ and all odd components of $G_{2}-B$ that are subgraphs of $F_{1}$ are also odd components of $G-B^{\prime}$. Obviously, $B^{\prime} \subsetneq X$.

Case 2: $C$ is 2-separation cut of $G_{2}$ that arise from 2-separation $\{s, z\}$, where $z \in \bar{X}$

Since every edge that joins a vertex of $F_{1}$ to $s$ is incident with $v$ in $G$, we have $\{v, z\}$ is a 2-separation of $G$, and $\partial\left(V\left(F_{1}\right)+v\right)$ is a 2-separation cut of $G$. Since $V\left(F_{1}\right)+v \subsetneq X, \partial\left(V\left(F_{1}\right)+v\right)$ does not cross $C$.

Now, we are going to prove our main result, Theorem 3.

Proof of Theorem 3 Let $C:=\partial(X)$ be a tight cut in a matching covered graph $G$ which is not an ELP-cut. We first prove the following two claims.

Claim 1: If $G$ has no barrier that is a proper subset of $X$ or $\bar{X}$, then there is a sequence $G_{1}=$ $G, G_{2}, \ldots, G_{r}, r \geq 2$ of matching covered graphs, such that for $i=1,2, \ldots, r-1, G_{i}$ has a 2-separation cut $C_{i}, G_{i+1}$ is a $C_{i}$-contraction of $G_{i}$, and $C$ is a 2-separation cut of $G_{r}$.

By Lemma 9, $G$ has a 2-separation cut $C_{1}$ that does not cross $C$. Let $G_{2}$ be the $C_{1}$-contraction of $G_{1}=G$ that contains $C$. By Lemma 7, $G_{2}$ has no nontrivial barrier that is a proper subset of one of two shores of $C$ in $G_{2}$. By Lemma 9, there is a 2-separation cut $C_{2}$ that does not cross $C$ in $G_{2}$. Applying Lemmas 7 and 9 repeatedly, we can recursively get $G_{i+1}$ is a $C_{i}$-contraction of $G_{i}$ for $i=1, \ldots$, where $C_{i}$ is a 2 -separation cut and does not cross $C$.

Since $V(G)$ is finite and $C$ is not an ELP-cut in $G$, there is an index $r$ such that $C$ is a 2-separation cut 
of $G_{r}$, where $r \geq 2$. Claim 1 holds.

Claim 2: If $G$ has a nontrivial barrier that is a proper subset of $\bar{X}($ resp. X), then $G$ has a nontrivial barrier cut $C^{*}$ such that one of its shores, say $Y$, is a superset of $X$ and $G^{\prime}:=G / \bar{Y}$ has no nontrivial barrier that is a proper subset of $V\left(G^{\prime}\right) \backslash X\left(\right.$ resp. $\left.V\left(G^{\prime}\right) \backslash \bar{X}\right)$.

Let $B$ be a nontrivial barrier of $G$. Without loss of generality, we assume that $B \subsetneq \bar{X}$. Since $G[X]$ is connected, there is an odd component $G[Y]$ of $G-B$ such that $X \subseteq Y$. Choose $B$ so that $|Y|$ is as small as possible. Let $G^{\prime}:=G / \bar{Y}$. Then $G^{\prime}$ has no nontrivial barrier that is a proper subset of $V\left(G^{\prime}\right) \backslash X$. Otherwise, we assume that $B^{\prime} \subsetneq V\left(G^{\prime}\right) \backslash X$ is a nontrivial barrier of $G^{\prime}$ and $G^{\prime}\left[Y^{\prime}\right]$ is an odd component of $G^{\prime}-B^{\prime}$ with $X \subseteq Y^{\prime}$. By Lemma 6, there is a nontrivial barrier $B^{\prime \prime} \subsetneq \bar{X}$ of $G$ such that $G\left[Y^{\prime}\right]$ is an odd component of $G-B^{\prime \prime}$. Obviously, $Y^{\prime} \subsetneq Y$, in contradiction to the minimality of $Y$. Hence Claim 2 holds.

If $G$ has no barrier that is a proper subset of $X$ or $\bar{X}$, the assertion holds by Claim 1 . If $G$ has a nontrivial barrier $B$ that is a proper subset of $X$ or $\bar{X}$, by symmetry, we may assume $B \subsetneq \bar{X}$. By Claim 2 , then $G$ has a nontrivial barrier cut $C^{*}:=\partial(Y)$ and $X \subsetneq Y$, and $G^{\prime}:=G / \bar{Y}$ has no nontrivial barrier that is a proper subset of $V\left(G^{\prime}\right) \backslash X$. Next if $G^{\prime}$ also has no nontrivial barrier that is a proper subset of $X$, then the assertion holds by Claim 1 . If $G^{\prime}$ has a nontrivial barrier that is a proper subset of $X$, by Claim 2 and Lemma 6, then $G^{\prime}$ has a nontrivial barrier cut $C^{\prime}:=\partial(Z)$ and $V\left(G^{\prime}\right) \backslash X \subsetneq Z$, and $G^{\prime \prime}:=G^{\prime} / \bar{Z}$ has no nontrivial barrier that is a proper subset of one of the two shores of $C$ in $G^{\prime \prime}$. By Claim 1, the assertion holds.

\section{Acknowledgements}

We would like to thank the responsible editor and the anonymous referees for their the constructive comments and kind suggestions on improving the representation of the paper.

\section{References}

J. Bondy and U. S. R. Murty. Graph Theory. Springer, New York, 2008.

M. H. Carvalho, C. L. Lucchesi, and U. S. R. Murty. On a conjecture of lovász concerning bricks. ii. bricks of finite characteristic. J. Combin. Theory Ser. B, 85(1):137-180, 2002.

M. H. Carvalho, C. L. Lucchesi, and U. S. R. Murty. On tight cuts in matching covered graphs. J. Comb., 9(1):163-184, 2018.

G. T. Chen, X. Feng, F. L. Lu, C. L. Lucchesi, and L. Z. Zhang. Laminar tight cuts in matching covered graphs. preprint, 2020. available at https: / / arxiv.org/abs/2003.08622.

J. Edmonds, L. Lovász, and W. R. Pulleyblank. Brick decompositions and the matching rank of graphs. Combinatorica, 2(2):247-274, 1982.

L. Lovász. Matching structure and the matching lattice. J. Combin. Theory Ser. B, 43:187-222, 1987.

M. D. Plummer. On n-extendable graphs. Discrete Mathematics, 31:201-210, 1980.

Z. Szigeti. Perfect matchings versus odd cuts. Combinatorica, 22:575-589, 2002. 